OPEN ACCESS

Edited by:

Shihua Zhang,

Academy of Mathematics and Systems Science - Chinese

Academy of Sciences, China

Reviewed by:

Xianwen Ren,

Institute of Pathogen Biology -

Chinese Academy of Medical

Sciences, China

Fabio lannelli,

FIRC Institute of Molecular Oncology,

Italy

${ }^{*}$ Correspondence: Guliang Wang

guliang.wang@austin.utexas.edu

Specialty section:

This article was submitted to Bioinformatics and Computational

Biology,

a section of the journal

Frontiers in Genetics

Received: 18 April 2016

Accepted: 15 July 2016

Published: 02 August 2016

Citation:

Wang $G$, Zhao J and Vasquez KM

(2016) Detection of cis-

and trans-acting Factors in DNA Structure-Induced Genetic Instability Using In silico and Cellular Approaches. Front. Genet. 7:135.

doi: 10.3389/fgene.2016.00135

\section{Detection of cis- and trans-acting Factors in DNA Structure-Induced Genetic Instability Using In silico and Cellular Approaches}

\author{
Guliang Wang *, Junhua Zhao and Karen M. Vasquez
}

Division of Pharmacology and Toxicology, College of Pharmacy, The University of Texas at Austin, Dell Pediatric Research

Institute, Austin, TX, USA

Sequences that can adopt alternative DNA structures (i.e., non-B DNA) are very abundant in mammalian genomes, and recent studies have revealed many important biological functions of non-B DNA structures in chromatin remodeling, DNA replication, transcription, and genetic instability. Here, we provide results from an in silico webbased search engine coupled with cell-based experiments to characterize the roles of non-B DNA conformations in genetic instability in eukaryotes. The purpose of this article is to illustrate strategies that can be used to identify and interrogate the biological roles of non-B DNA structures, particularly on genetic instability. We have included unpublished data using a short $\mathrm{H}$-DNA-forming sequence from the human $c-M Y C$ promoter region as an example, and identified two different mechanisms of H-DNAinduced genetic instability in yeast and mammalian cells: a DNA replication-related model of mutagenesis; and a replication-independent cleavage model. Further, we identified candidate proteins involved in $\mathrm{H}$-DNA-induced genetic instability by using a yeast genetic screen. A combination of in silico and cellular methods, as described here, should provide further insight into the contributions of non-B DNA structures in biological functions, genetic evolution, and disease development.

Keywords: non-B DNA, triplex, genetic instability, replication, DNA repair

\section{INTRODUCTION}

Our understanding of the structure and function of genomic DNA has been dramatically advanced in the past few decades. More than $50 \%$ of genomic DNA contains repetitive sequences that do not necessarily code for protein (Lander et al., 2001), and were previously regarded as "junk DNA" (Lewin, 1986; Veitia and Bottani, 2009). In the past several decades, much research has supported the notion that these non-coding repetitive sequences play important biological roles in cells. In addition to the regulatory functions of non-coding RNAs (ncRNAs) such as microRNAs (miRNAs) that are transcribed from these "junk DNA" sequences (Perera and Ray, 2012; Hausser and Zavolan, 2014), many repetitive sequences are involved in chromosome architecture and organization and gene expression regulation due to their ability to adopt DNA conformations that differ from the canonical B-form DNA (i.e., non-B DNA). 


\section{Non-B DNA Structure Formation at Repetitive Regions in the Genome}

There are many published reports on the functions of non- $B$ DNA-forming sequences in the regulation of gene expression, DNA replication, recombination, and telomere maintenance (van Holde and Zlatanova, 1994; Parkinson et al., 2002; Hanawalt and Spivak, 2008; Jain et al., 2008; Wang and Vasquez, 2009; Bochman et al., 2012; Boyer et al., 2013; Bansal et al., 2014). More than 15 types of alternative non-B DNA structures have been described to date (Choi and Majima, 2011; Wang and Vasquez, 2014); for example, simple repeats can form small "loop-out" bubbles caused by the misalignment of the repeat units (Mirkin, 2007), trinucleotide repeats can form imperfect hairpin structures, and inverted repeat sequences have the potential to adopt perfect self-complementary hairpins and cruciforms (Sinden et al., 1991; Voineagu et al., 2008; Lu et al., 2015). A ZDNA structure with a left-handed zigzag-shaped backbone can form at alternating purine-pyrimidine regions such as GC or GT repeats, with the latter repeat representing the most abundant dinucleotide repeat in the human genome (Rich and Zhang, 2003). Z-DNA structures have also been demonstrated to form on CCG/CGG repeats in the presence of aluminum ions (Latha et al., 2002). Intramolecular triplex structures (HDNA) can form at mirror-repeat homopurine/homopyrimidine sequences via formation of Hoogsteen hydrogen bonding of the purine-rich strand through the major groove of the underlying duplex (Voloshin et al., 1988; Frank-Kamenetskii and Mirkin, 1995). Because four guanine bases can associate through Hoogsteen-hydrogen bonding to form a square planar structure (guanine tetrad), four guanine-rich regions (from the same DNA molecule or different molecules) that contain runs of three or more guanines can stack to form G-quadruplex or G4 DNA structures (Lane et al., 2008; Bochman et al., 2012).

\section{Identification of Genomic cis Elements with the Potential to Adopt Non-B DNA Structures}

The formation of non-B DNA structures requires appropriate repetitive sequence elements (as mentioned above), which allows the development of computational algorithms to search for genomic segments that have the potential to adopt non- $B$ DNA structures. Many such in silico search algorithms are available on-line and can be used as a first step to uncover the biological functions of non-B DNA. For example, "einverted"1 or "palindrome" can be used to identify potential hairpin or cruciform-forming inverted repeats; "QGRS Mapper" searches for potential quadruplex-forming sequences ${ }^{3}$ (Kikin et al., 2006); and Cer et al. (2013) and our group have published algorithms to search for potential H-DNA-forming and Z-DNA-forming

\footnotetext{
${ }^{1}$ http://emboss.bioinformatics.nl/cgi-bin/emboss/einverted

${ }^{2}$ http://emboss.bioinformatics.nl/cgi-bin/emboss/palindrome

${ }^{3} \mathrm{http} / / /$ bioinformatics.ramapo.edu/QGRS/index.php
}

sequences $^{4}$ (Wang et al., 2013) and Non-B DB v2.0 (Cer et al., 2013).

\section{Non-B DNA and Genetic Instability Hotspots}

An important discovery in the field of DNA structure is that many types of non-B DNA structures can lead to genetic instability in prokaryotic and eukaryotic cells in the absence of exogenous DNA damage (Wells et al., 2005; Wang and Vasquez, 2006; Zhao et al., 2010; Du et al., 2014; Lu et al., 2015). Genetic instability, from point mutations that change single base pairs to massive chromosomal aberrations, is a hallmark of many human diseases, including cancer. Thus, great effort has been expended to elucidate the mechanisms involved in DNA structure-induced genetic instability. Most models of genetic instabilities assume that DNA damage and mutation occur randomly, and those that confer survival/growth advantages are selected for, allowing continuous adaptation of tumor cells from normal tissue (Caporale, 2003; Wreesmann and Singh, 2005; Venkatesan et al., 2006). However, accumulating evidence provided by DNA sequencing of human cancer genomes (Sinclair et al., 2011) indicates that mutations are not distributed randomly in genomes. Common disease-associated "hotspots" where nonrandom mutations cluster have been reported in human genomes (Cleary and Sklar, 1985; Montoto et al., 2003; Popescu, 2003; Mefford and Eichler, 2009). Inspection of the sequences at or near those genetic instability hotspots has revealed that many non-B DNA-forming sequences are enriched at these regions, suggesting a correlation between non-B DNA structure-forming sequences and disease-associated genetic instability (Bacolla and Wells, 2004; Bacolla et al., 2004; Choi and Majima, 2011; Chen et al., 2013; Kamat et al., 2016). Computational analysis of human cancer DNA sequencing databases coupled with nonB DNA search algorithms can further reveal the connection between cis-acting non-B DNA-forming elements and genetic instability in human disease. Using this strategy, we have recently found that non-B DNA-forming sequences were significantly enriched within \pm 200 bp of breakpoints characterized in 19,947 translocations and 46,365 deletions in human cancer genomes, such as simple AT repeats that can form slippage structures or cruciform/hairpin structures, and GAA or GAAA repeats that can form slippage structures or H-DNA structures, and non-simple repetitive sequences that form H-DNA and Z-DNA conformations (Bacolla et al., 2016). We have also reported that short cruciform-forming inverted repeats are significantly enriched at translocation breakpoint hotspots in cancer genomes by searching within $\pm 100 \mathrm{bp}$ surrounding the translocation hotspots in $\sim 20,000$ human cancer genomes (Lu et al., 2015). Together, with findings that these non-B DNA structures are mutagenic in mammalian cells (Wang and Vasquez, 2004; Wang et al., 2006; Lu et al., 2015), these results clearly suggest that nonB DNA-forming sequences represent an intrinsic risk factor for genomic instability hotspots in human diseases.

\footnotetext{
${ }^{4}$ http://sites.utexas.edu/adrgs/research-tools/dna-structure-search/

${ }^{5}$ http://nonb-abcc.ncifcrf.gov
} 


\section{Non-B DNA-Forming Sequences Co-localize with Genetic Instability "Hotspots" in Human Cancer}

Here we used two oncogenes, $c-M Y C$ and $B C L-2$, as examples of co-localization of non-B DNA-forming sequences with genetic instability hotspots, because they are commonly involved in chromosomal translocations and the breakpoints in human cancer characterized. We analyzed a short 500-bp region from the human $c-M Y C$ promoter between $\mathrm{P} 0$ and $\mathrm{P} 1$ that represents a major breakage hotspot found in $c-M Y C$ translocation-induced lymphomas and leukemia (Care et al., 1986; Haluska et al., 1988; Joos et al., 1992; Saglio et al., 1993; Wilda et al., 2004). DNA double-strand breaks (DSBs) and subsequent translocation events can position the $c-M Y C$ gene under the control of a strong promoter of an immunoglobulin gene, leading to activation and overexpression of the oncogene in certain cancers (Care et al., 1986; Ramiro et al., 2006). H-DNA (Mirkin et al., 1987), Z-DNA (Rimokh et al., 1991; Wolfl et al., 1995) and G-quadruplex-forming sequences (Siddiqui-Jain et al., 2002) have been identified near this translocation breakage hotspot region. Using our non-B DNA search algorithm (Wang et al., 2013), we systematically inspected the $c-M Y C$ and $B C L$ 2 genes that are often involved in translocations in human cancers, for potential H-DNA- and Z-DNA-forming sequences (Figure 1). For H-DNA (shown on the left), we searched for homopurine/homopyrimidine sequences that contained mirrorrepeat symmetries of a minimum repeat arm of $6 \mathrm{bp}$, and a 1-12 bp "spacer" sequence between the two mirror repeats. We allowed for one mismatch in symmetry when the "arms" were $\geq 10 \mathrm{bp}$, since a longer spacer between two mirror repeats or more mismatches could reduce the stability of an H-DNA structure. For Z-DNA (shown on the right), we searched for alternating purine-pyrimidine sequences that have a Z-DNA score of $\geq 75$ [briefly, in contiguous alternating purine/pyrimidine fragments, each GC dinucleotide has a score of 25 and each GT or CA dinucleotide has a score of 3 . For more details on the non-B DNA search algorithm and parameters for H-DNA and Z-DNA searches, please see (Wang et al., 2013)]. Figure 1A shows the search report of human $c-M Y C$ gene from the promoter to exon 1 . Chromosome breakpoints involved in $c$-MYC translocations in human Burkett's lymphoma and mouse plasmacytomas, or single-strand specific S1 nuclease sensitive sites in cultured human cells (Care et al., 1986; Haluska et al., 1988; Joos et al., 1992; Saglio et al., 1993; Michelotti et al., 1996; Kovalchuk et al., 1997; Wilda et al., 2004) are marked as red stars in Figure 1A. All previously reported H-DNA- forming (Mirkin et al., 1987) and Z-DNA-forming sequences (Wittig et al., 1992) identified by in vitro experiments were identified in the search, and were located on the $c-M Y C$ gene (Figure 1A). Figure 1B shows the search result of H-DNA forming sequences in the first 70 bps of the human BCL-2 major breakpoint region (left), and the chromosome breakpoints within this area that are involved in BCL-2 translocations (right, marked as red stars; Wyatt et al., 1992; DiCroce and Krontiris, 1995; Jager et al., 2000; AlbingerHegyi et al., 2002; Raghavan et al., 2004). The co-localization of non-B DNA-forming sequences and chromosomal breakpoints identified in the $c-M Y C$ and $B C L-2$ genes in translocation-related cancers (Figure 1), and a genome-wide significant enrichment of non-B DNA-forming sequences at genomic breakpoints in human cancer (Bacolla et al., 2016) suggest a role for non-B DNA in genetic instability and cancer development.

\section{Mechanisms of H-DNA-Induced Genetic Instability}

DNA sequences that have the potential to adopt non-B DNA structures have been shown to impact DNA replication, transcription, DNA damage and DNA repair by recruiting and interacting with trans-acting proteins that bind, stabilize/destabilize or process non-B DNA conformations. Thus, identification of the proteins that interact with non-B DNA is critical to elucidate the mechanisms involved in non-B DNA-induced genetic instability. Because strategies to directly identify non-B DNA interacting proteins can be technically challenging, most approaches to date are based on functional analysis, i.e., by comparing the outcome of a non-B DNA structure-induced phenotype (mutagenesis) in cell lines/animals that are deficient or proficient in a gene of interest. Therefore, a fast, versatile and sensitive system to detect non-B DNA-induced genetic instability is warranted.

We have used a series of different mutation-reporter systems that can detect a variety of genetic instability events including base substitutions, point mutations, small and large deletions and insertions, and DSBs in bacteria, cultured mammalian cells, yeast and mice (Wang et al., 2009) and found that many different types on non-B DNA including H-DNA, Z-DNA and cruciform structures can stimulate genetic instability (Wang and Vasquez, 2004; Wang et al., 2006, 2008; Lu et al., 2015). Using these mutation-reporter systems, we have identified at least two different mechanisms of non-B DNA-induced mutagenesis: a DNA replication-dependent mechanism where the non-B DNA structure impacts the replication machinery, resulting in slippage and misalignment events at simple repeats, and replication fork stalling and collapse that can lead to DSBs; and a DNA structurespecific cleavage model that does not require DNA replication, in which the non-B DNA structures may be recognized as "damage" and cleaved by the DNA repair machinery (Wang and Vasquez, 2009, 2014; Vasquez and Wang, 2013). These models are discussed in more detail below.

\section{Replication-Related Mechanisms of H-DNA-Induced Genetic Instability}

To determine if H-DNA acts as an impediment to DNA replication, we measured replication fork stalling at a short H-DNA-forming sequence by using 2-D gel electrophoresis analysis and Southern blotting. The plasmid pMYC contains a short H-DNA-forming sequence from the human $c-M Y C$ promoter as identified in Figure 1 (located at -100; Wang and Vasquez, 2004), while pCON contains a control B-DNA sequence. The plasmids were transfected into mammalian COS7 cells and the replication intermediates of were recovered $24 \mathrm{~h}$ later, followed by $D p n I$ digestion to remove unreplicated DNA. The NdeI and BsaI fragments containing the H-DNA or 
A

8

\begin{tabular}{|l|r|l|}
\hline \multicolumn{1}{|c|}{$\boldsymbol{H} \boldsymbol{\nabla}$} & $\begin{array}{r}\text { Start }^{5} \text { in } \\
\text { Gene } \boldsymbol{\Lambda} \boldsymbol{\nabla}\end{array}$ & Region $^{5}$ \\
\hline GGAAAGAGGACTGGAAAGG & -1210 & putative-promoter \\
\hline GGAGGAATGATAGAGGCATAAGGAGG & -529 & putative-promoter \\
\hline AGGGAGAGGGTTTGAGAGGGA & -265 & putative-promoter \\
\hline GGAGGGTGGGGAAGGTGGGGAGG & -126 & putative-promoter \\
\hline GGGAGGGTGGGGAGGG & -116 & putative-promoter \\
\hline GGGAGGGGCGCTTATGGGGAGGG & -100 & putative-promoter \\
\hline GGGAGAGGAGAAGGCAGAGGG & 750 & intron_1 \\
\hline AGGGGATAGCTCTGCAAGGGGA & 2058 & intron_1 \\
\hline
\end{tabular}

\begin{tabular}{|c|c|c|}
\hline H-DNA & $\Delta \nabla$ & $\begin{array}{l}\text { Start }{ }^{3} \text { in } \\
\text { Sequence } \Delta \nabla\end{array}$ \\
\hline AGGAAGACCCT GAAGGA & & 193100 \\
\hline GAAGGACAGCCATGAGAAAG & & 193089 \\
\hline GAGAAAGCCCCCGCGGAAGGAG & & 193076 \\
\hline GGAAGGAGGGCAGGAGGG & & 193062 \\
\hline GGAAGGAGGGCAGG & & 193062 \\
\hline AGGAGGGCAGGAGGG & & 193059 \\
\hline GGAGGGCTCTGGGTGG & & 193050 \\
\hline
\end{tabular}
$\left\lceil\quad \begin{array}{l}\mid \overrightarrow{0} \\ \text { वे }\end{array}\right.$

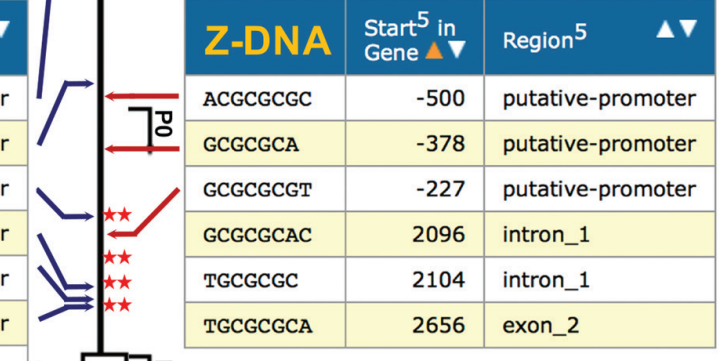

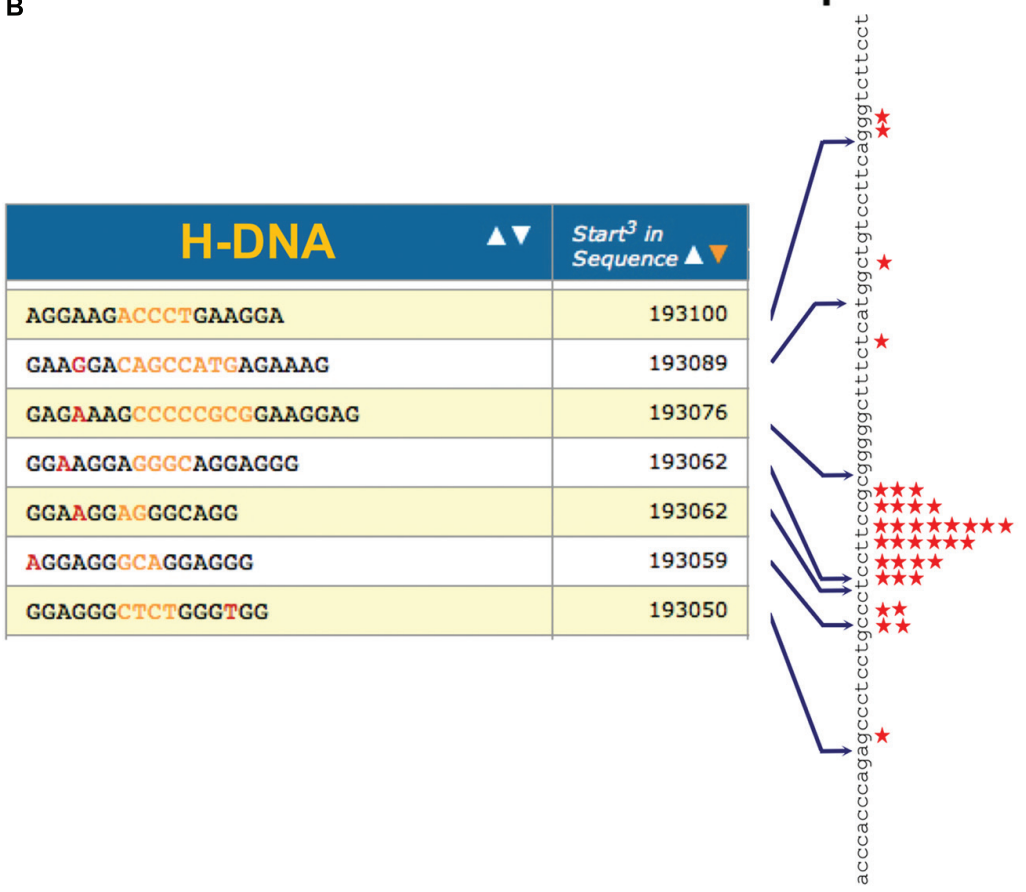

FIGURE 1 | Enrichment of non-B DNA structure at human mutation hotspots. (A) Co-localization of H-DNA- and Z-DNA-forming sequences with mutation hotspots in the human c-MYC promoter. Search results for H-DNA- or Z-DNA-forming sequences in the promoter region of the human $c-M Y C$ gene are shown. PO, $\mathrm{P} 1$ and P2, Exon 1, and Intron 1 of the human $c-M Y C$ gene are shown in the center. Potential H-DNA-forming sequences are shown on the left and potential Z-DNA-forming sequences are shown on the right. Chromosome breakpoints involved in c-MYC translocations in previous reports, or single-strand-specific S1 nuclease sensitive sites in cultured human cells are marked as red stars. (B) Co-localization of $\mathrm{H}$-DNA-forming sequences within the human $B C L-2$ major breakpoint region. Search results for $\mathrm{H}$-DNA-forming sequences in the upstream 70-bp from major breakpoint region of the human BCL-2 gene are shown on the left. Chromosome breakpoints involved in BCL-2 translocations in previous reports are marked as red stars on the right. 
control inserts were separated by mass via gel electrophoresis in the first dimension, and by mass and shape by agarose gel electrophoresis in the second dimension, resulting in a typical Y-shape replication arc. As shown in Figure 2, H-DNA indeed stalled DNA replication, resulting in a bulge on the right arm of the arc and a much lighter left arm of the replication arc indicating stalling at/near the H-DNA insert resulting in fewer replication intermediates past the $\mathrm{H}-\mathrm{DNA}$ sequence. In contrast, the control pCON plasmid formed a much smoother replication arc with near equal density of the left and right arms, indicating continuous replication. This result suggests that $\mathrm{H}$-DNA can stall or pause the DNA replication machinery in vivo, perhaps in a fashion similar to our previous observation of H-DNAinduced RNA polymerase arrest within/near and downstream of the H-DNA-forming sequence (Belotserkovskii et al., 2007). These results are also consistent with our previous demonstration that this H-DNA-forming sequence can stimulate the formation of DSBs in an area $\sim 100$-bp up-stream and down-stream to the H-DNA structure (Wang and Vasquez, 2004). Thus, replication stalling-related DNA breakage is a likely mechanism for genetic instability induced by H-DNA in mammalian cells.

\section{H-DNA-Induced Genetic Instability Independent of DNA Replication}

Using same mutation-reporter shuttle vector as described above, we further investigated whether DNA replication was necessary for H-DNA-induced mutagenesis in mammalian cells. The

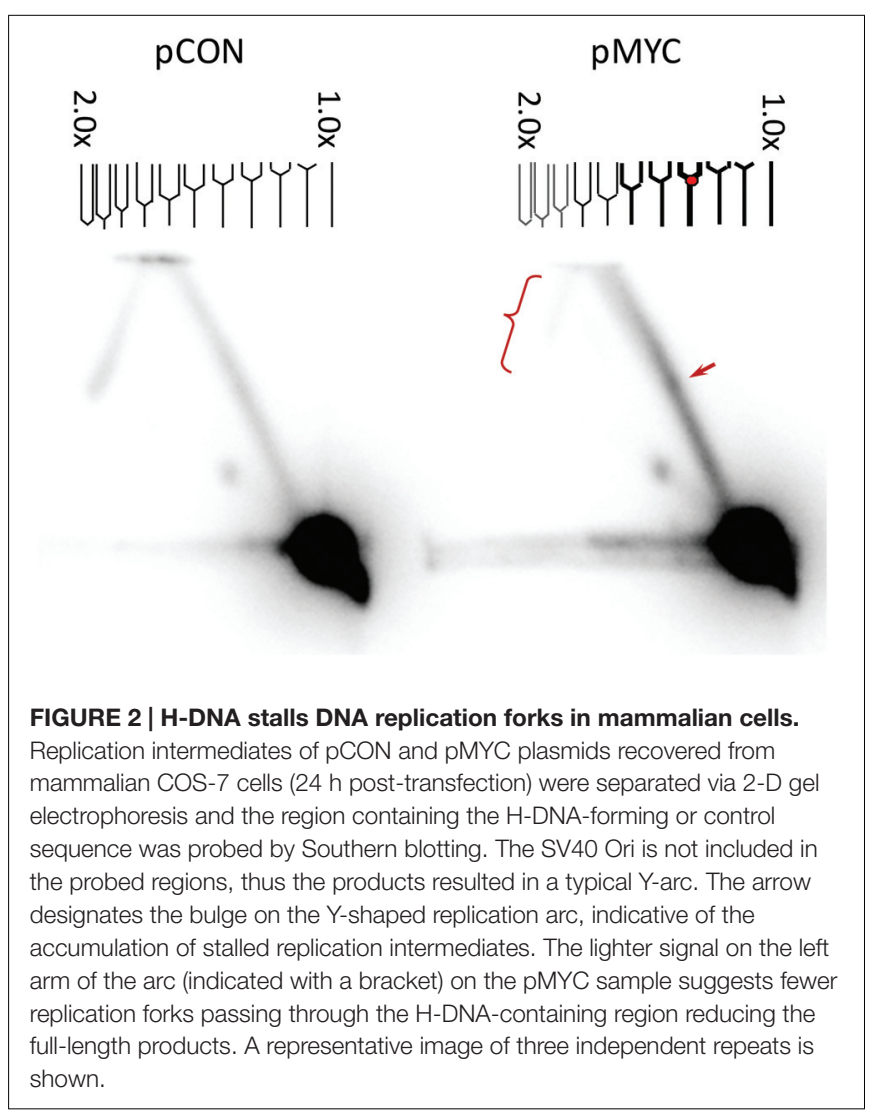

SV40 replication origin on the vector allows control of the plasmid DNA replication, which for these studies provides an advantage over using endogenous genomic DNA elements. Here, we incubated the plasmids, pMYC and pCON, with HeLa cell free extract for $6 \mathrm{~h}$. Plasmid DNA replication occurred only when the extract was supplemented with the SV40 T antigen. Interestingly, H-DNA stimulated mutations in both replicationcompetent and replication-incompetent extracts (Figure 3A). Although, direct comparison of the H-DNA-induced mutation frequencies in the replication-proficient and replication-deficient systems cannot be made (because the reporter plasmids recovered from the replicating system were digested with $D p n I$ to remove the unreplicated DNA), this result indicated that H-DNA can stimulate genetic instability independent of DNA replication, similar to our results with Z-DNA- and cruciform-induced mutagenesis as we have previously reported (Wang et al., 2006; Lu et al., 2015).

To determine the spectrum of DNA breaks induced by $\mathrm{H}$-DNA in the absence of replication, we radiolabeled the $5^{\prime}$-ends of the breakpoints on the plasmids incubated with the replication-incompetent HeLa cell extract and separated the $\sim 400$ bp BsaI-BamHI restriction fragments containing the H-DNA or control inserts by agarose gel electrophoresis. As shown in Figure 3B, all H-DNA-containing plasmids, pMYC, pGG32 and pAG32 plasmids (Wang and Vasquez, 2004) exhibited full-length restriction fragments and shorter fragments, indicating that both DNA SSBs and DSBs were generated near the H-DNA structure. These breakpoints were not seen in the plasmids that were incubated in the absence of cell extract, as expected. This result clearly suggested a replication-independent cleavage activity at/near the H-DNA structure that might be responsible, in part, for the mutagenesis observed in Figure 3A.

\section{Identification of trans-acting Factors Involved in H-DNA-Induced Mutagenesis}

To identify the cis-acting factors involved in non-B DNA-induced genetic instability, we utilized a yeast artificial chromosome (YAC) to screen a yeast mutant library (Lu et al., 2015). The YAC-based reporter system provides a facile screening strategy in a eukaryotic system. It has advantages over the plasmidbased approach in that the non-B DNA sequence is integrated in a chromosomal context, and that knock-out yeast libraries are commercially available that allow complete depletion of the genes of interest (compared with an siRNA-depletion approach in human cells). The system we used here contains H-DNA (or a B-DNA control sequence) integrated between a URA3reporter gene and a C4A4 telomere seed sequence to rebuild the telomere after loss of the distal arm in the YAC (Wang et al., 2009; Lu et al., 2015), which allows for detection of non-B DNA-induced DSBs (Wang et al., 2009; Lu et al., 2015). H-DNAinduced DSBs will result in the loss of URA3, which can be selected for by growth in medium containing 5-Fluoroorotic Acid (5-FOA). As shown in Table 1, in wild-type (WT) yeast BY4742 cells, a short H-DNA-forming sequence from the human $c$-MYC promoter induced $\sim 9.4$-fold more FOA-resistant URA3colonies over that of the control, consistent with our findings 
A

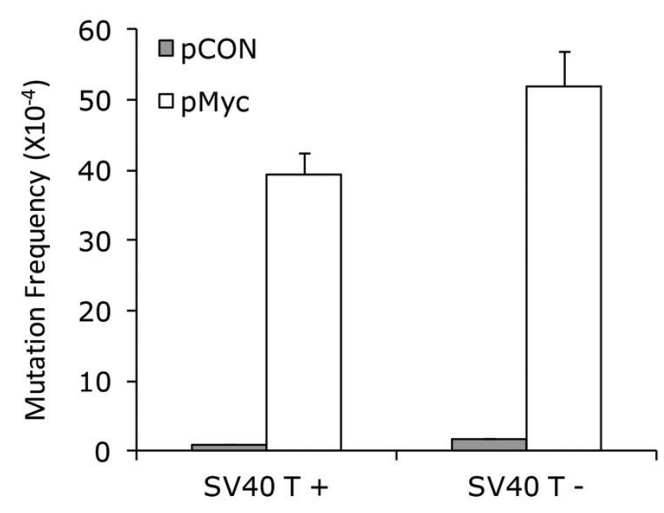

\section{B}

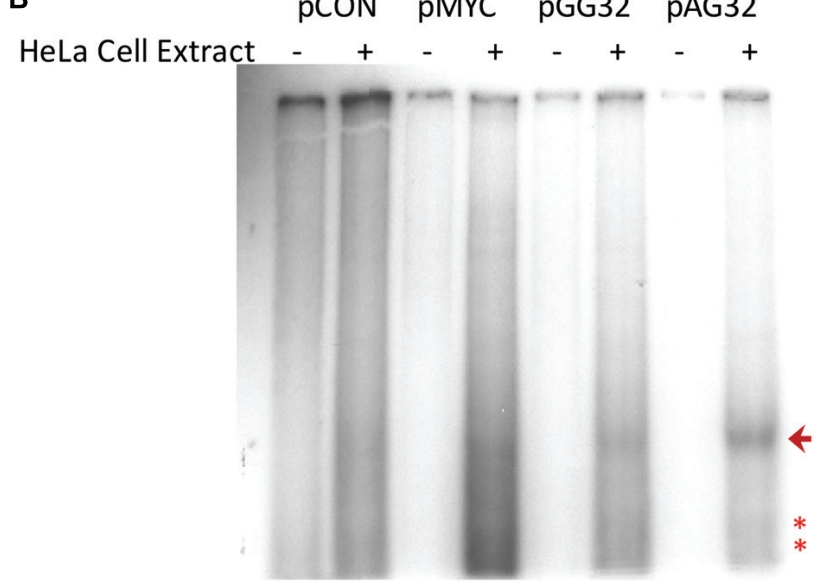

FIGURE 3 | Replication-independent H-DNA-induced genetic instability in HeLa cell extracts. (A) H-DNA-induced mutation frequencies in the presence (SV4OT+) or absence (SV4OT-) of replication in HeLa cell-free extracts. The number of mutants observed and the total number of colonies screened are listed below the corresponding bars. Error bars show the standard errors of the mean value of three independent experiments. (B) $\mathrm{H}$-DNA-induced cleavage in replication-incompetent HeLa cell extracts. A radiolabeled 400-bp fragment containing SSBs generated in HeLa cell extracts ( + ) is indicated by an arrow, and the radiolabeled shorter fragments indicative of H-DNA-induced DSBs in HeLa cell extracts are marked with "*". Plasmids incubated in the absence of HeLa cell extracts (-) served as controls.

TABLE 1 | An H-DNA-forming sequence induces DNA instability in WT and deficient yeast.

\begin{tabular}{|c|c|c|c|c|c|}
\hline & Control* & H-DNA* & & F** & \\
\hline BY4742(WT) & 1.24 & 11.6 & & & 9.35 \\
\hline $\operatorname{rad} 50 \Delta$ & 2.28 & 14.33 & & 6.29 & \\
\hline mre11 $\Delta$ & 1.78 & 2.33 & 1.31 & & \\
\hline sae2 $\Delta$ & 6.4 & 18.4 & 2.88 & & \\
\hline exols & 0.95 & 7.96 & & & 8.38 \\
\hline yen/s & 5.28 & 13.8 & 2.61 & & \\
\hline topo 1 & 0.17 & 1.8 & & & 10.59 \\
\hline topo3 & 6.33 & 20.37 & 3.22 & & \\
\hline$i s w / \Delta$ & 2.92 & 11.28 & 3.86 & & \\
\hline
\end{tabular}

*Number of yeast colonies with loss of Ura3 in 100,000 cells. At least 30,000 colonies were screened in each fragility assay.

${ }^{* *} \mathrm{H} / \mathrm{C}$ represents the fold increase in genetic instability induced by $\mathrm{H}$-DNA over control in the various yeast strains. H-DNA-induced significantly more mutants in all WT and deficient yeast strains. P-values $<0.05$ in two-sided t-test comparing $H$-DNA vs. Control, from $>3$ repeats. H-DNA-induced mutation above control in all deficient yeast strains list above are significantly different from that from WT cells. $P$-values $<0.05$ in Fisher's exact test from $>3$ repeats.

from mutation-reporter plasmids in mammalian cells (Wang and Vasquez, 2004). The same YAC reporter constructs were then transformed into various mutant yeast strains deficient in genes of interest via a kar-cross method (Wang et al., 2009; Lu et al., 2015) and H-DNA-induced genetic instability was measured. Results from an initial screen are listed in Table 1.

As we have reported previously, H-DNA can stimulate DSBs in mammalian cells, with the vast majority of mutations being largescale deletions containing microhomologies at the junctions, suggesting a role for a micro-homology-mediated end-joining (MMEJ) mechanism in generating H-DNA-induced mutations in vivo (Wang and Vasquez, 2004). Thus, we tested H-DNAinduced genetic instability in yeast strains deficient in proteins involved in such pathways. We found that deletion of mre11 diminished the H-DNA-induced mutagenesis to background levels (only 1.3-fold above the control sequence in mre11 $\Delta$ ), suggesting that Mre11 is involved in the H-DNA-induced mutagenesis (Table 1). Yeast strains deficient in rad50 and sae2 also reduced the H-DNA-induced genetic instability, though not as dramatically as in the mre $11 \Delta$ cells, suggesting roles for these proteins in H-DNA processing and H-DNA-induced mutations. The Mre11-Rad50 complex has been shown to cleave hairpin structures at the junction of the center loop and stem, and the duplex stem and the $3^{\prime}$ single-strand overhang (Trujillo and Sung, 2001), indicating a ssDNA-dsDNA junction-specific activity. Sae2 is an endonuclease that interacts with the Mre11-Rad50-Xrs2 (MRX) complex in processing hairpin DNA structures during DSB repair (Lobachev et al., 2002). Thus, it is possible that similar ssDNA-dsDNA junctions found in H-DNA could be recognized and cleaved by this protein complex, leading to genetic instability. Similarly, exo1 that encodes a $5^{\prime}-3^{\prime}$ exonuclease and shares functional overlap with Mre11 and Rad27 nucleases in DNA metabolism (Moreau et al., 2001), might also be involved $\mathrm{H}$-DNA-induced mutations, as indicated by the reduced level of $\mathrm{H}$-DNA-induced genetic instability in the exo1 $\Delta$ cells (Table 1).

We utilized the YAC screen in yeast deficient in enzymes that are involved in other DNA processes, for example, Yen1 Holliday junction resolvase, which is the homolog of human GEN1. Overexpression of GEN1 was reported to resolve the accumulated cruciform-shaped replication intermediates at damaged forks caused by Sgs1-deficiency in yeast cells (Mankouri et al., 2011). We found that deficiency of yen 1 reduced the level of H-DNAinduced mutagenesis in our YAC-reporter system, suggesting a potential role in resolving H-DNA structures. However, further 
work is warranted to determine if Holliday junction resolvases can cleave the branched structure on H-DNA and contribute to genetic instability.

Topoisomerases can relax the negative supercoiling on duplex DNA (Wang, 2002), which might assist in resolving non-B DNA conformations, including H-DNA. Consistent with this notion, we found that topo1 deficiency, one of the major nuclear enzymes that relieves torsional strain in DNA, led to a modest increase in H-DNA-induced genetic instability. Since topoisomerases have been shown to cleave non-B DNA structures (Pognan and Paoletti, 1992; Froelich-Ammon et al., 1994), genetic instability could result if the breakpoints were not faithfully rejoined. Here we found that deficiency of topo3 that codes for a conserved protein (in a complex with Sgs1 and Rmi1) that is primarily implicated in homologous recombination and disassembly of branched DNA structures (Mankouri and Hickson, 2007; De Muyt et al., 2012) also contributed to H-DNA-induced genetic instability (Table 1).

The formation of non-B DNA structures is a dynamic process and is affected by many factors including chromatin-remodeling proteins. The ISW1 gene codes for an ATPase subunit of the largest class of mammalian remodelers ISWI (Goldman et al., 2010) and is involved in transcription elongation (Morillon et al., 2003; Smolle et al., 2012). We found that the absence of isw1 substantially reduced the H-DNA-induced genetic instability compared to WT yeast cells. Future studies are warranted to determine the extent to which the direct chromatin remodeling function and/or the transcription elongation function of ISWI led to this effect.

The field of DNA structure-induced genetic instability is still at an early stage and much more work is required to determine the mechanisms involved for each type of non-B DNA structure. The gene products identified in the YAC screening system still need to be confirmed in other systems (e.g., mammalian cell systems or mouse models), and the mechanisms of how these proteins are involved in H-DNA formation and processing need to be explored. Updated databases of mutation hotspots in human disease, coupled with information on DNA modifications, epigenetics, and chromosome structure, will allow for further advances to be made in understanding how non-B DNA-forming sequences impact disease etiology. New methods to detect nonB DNA conformations, particularly in living cells, will facilitate the elucidation of the biological functions of these non-B DNA structures.

\section{MATERIALS AND METHODS}

\section{In vitro Mutagenesis Assay in HeLa Cell-free Extracts}

The mutation-reporter plasmids contain an SV40 origin of replication and can only be replicated in the presence of the SV40 large $\mathrm{T}$ antigen. $50 \mathrm{ng}$ of plasmid DNA was incubated with $180 \mu \mathrm{g}$ SV40 large T antigen-deficient HeLa cell-free extract from a CHIMERx DNA replication assay kit (Milwaukee, WI, USA) supplied with $1 \mu \mathrm{g}$ SV40 large $\mathrm{T}$ antigen (for the replication-competent system) for $6 \mathrm{~h}$ at $37^{\circ} \mathrm{C}$ in a reaction buffer (30 mM HEPES, $\mathrm{pH} 7.5,7 \mathrm{mM} \mathrm{MgCl}_{2}, 0.5 \mathrm{mM}$ dithiothreitol, $4 \mathrm{mM}$ ATP, $100 \mu \mathrm{M}$ each of dNTP, $50 \mu \mathrm{M}$ each of NTP, $40 \mathrm{mM}$ phosphocreatine, 0.625 units of creatine phosphokinase). Purified large T antigen (CHIMERx) was added to the manufacturer's recommended reaction mixture to allow for DNA replication. H-DNA-induced mutants were determined by blue-white screening and analyzed by restriction digestion analysis and DNA sequencing as we have described (Wang and Vasquez, 2006).

\section{In vitro H-DNA-Induced Incision Assay in HeLa Cell Extracts}

Fifty ng of plasmid DNA pCON, pMYC, pGG32 or pAG32 was incubated with $180 \mu \mathrm{g}$ HeLa cell-free extract as in the replication assay (see above) for $4 \mathrm{~h}$ in the absence of SV40 large T antigen. After purification, the $5^{\prime}$-ends produced at the breakpoints in HeLa extract were radiolabeled with T4 polynucleotide kinase and $\left[\gamma-{ }^{32} \mathrm{P}\right]$ ATP and then the plasmids were digested with $B s a I$ and BamHI to release an $\sim 400$-bp fragment containing the H-DNA or control insert. Products were separated on $1.5 \%$ agarose gels and subjected to autoradiography. Plasmids incubated under similar conditions but in the absence of HeLa cell extract were included as controls.

\section{Two-Dimensional Gel Electrophoresis Analysis of Replication Intermediates in Mammalian Cells}

Plasmids were transfected into mammalian COS-7 cells using GenePORTER (GTS, Inc., San Diego, CA, USA) according to the manufacturer's recommendations. After $24 \mathrm{~h}$, replication intermediates were isolated and subjected to $2 \mathrm{D}$ gel electrophoresis according to Krasilnikova and Mirkin (2004). After being transferred to membranes, replication intermediates were identified by radiolabeled probes specific to this NdeI-BsaI fragment.

\section{Yeast Strains and YACs}

Yeast strain 213 (MATa Kar1-1 Leu2-3,112 Ura3-52 his7), was used for investigation of the mutagenic capability of the $\mathrm{H}$-DNA sequences and for kar-crossing with other yeast strains to introduce the reporter YACs. Yeast strain BY4742 (MAT $\alpha$, his $3 \Delta 1$, leu $2 \Delta 0$, lys $2 \Delta 0$, ura $3 \Delta 0$ ) and its derivatives were used to determine the mutation frequency variance between the WT strain and the mutant strains. YACs containing the H-DNA or control sequences were constructed by cloning these sequences between the C4A4 and URA3 genes on a replication-defective pRS306 plasmid. AatII linearized plasmids were introduced into a URA3- host strain containing a YAC with a non-functional point-mutated URA3 gene. Homologous recombination between YAC VS5 and the pRS306 plasmid generated a YAC containing the insert and a functional URA3 gene. After transformation, $\mathrm{URA} 3+$ transformants were selected on SD+DO-Leu-Ura plates and the presence of $\mathrm{H}$-DNA or the control B-DNA sequence on the YAC was confirmed by colony PCR followed by direct DNA sequencing, as previously described (Lu et al., 2015). 


\section{YAC Instability Assay}

Donor cells K213 (MATa Kar1-1, his7, leu2-3, 112, ura3-52) containing YACs with the H-DNA-forming sequence or the control B-DNA-forming sequence were grown from a single colony overnight at $30^{\circ} \mathrm{C}$. Canavanine (Sigma) resistant recipient cells (BY4742 background, MATa his3- $\Delta 1$, leu2- $\Delta 2$, ura3- $\Delta 0$, ATCC) from the deletion library (GSA-5, ATCC, Manassas, VA, USA) were grown in YEPD at the permissive temperature overnight. YACs were transferred from donor cells to recipient cells via kar-crossing and were selected on SD+DO-UraArg+canavanine $(60 \mathrm{mg} / \mathrm{L})$ plates. The YAC transfer was confirmed by survival on SD+DO-Ura-Leu plates, but not on $\mathrm{SD}+\mathrm{DO}$-Lys plates. Insertion sequences on the YACs were further confirmed by PCR amplification. Five (5) starting colonies were used to inoculate cultures that were grown overnight at the permissive temperature in SD+DO-Leu. $50 \mu \mathrm{l}$ of each culture was plated on SD+DO-Leu with 5-fluoroorotic acid (5-FOA, Zymo Research, Irvine, CA, USA) plates to select for breakage events, while another $50 \mu \mathrm{l}$ of each culture was combined, and the mixture was diluted and plated on SD+DOLeu plates for a total cell number count. The mutation frequency

\section{REFERENCES}

Albinger-Hegyi, A., Hochreutener, B., Abdou, M. T., Hegyi, I., DoursZimmermann, M. T., Kurrer, M. O., et al. (2002). High frequency of $\mathrm{t}(14 ; 18)$-translocation breakpoints outside of major breakpoint and minor cluster regions in follicular lymphomas: improved polymerase chain reaction protocols for their detection. Am. J. Pathol. 160, 823-832. doi: 10.1016/S00029440(10)64905-X

Bacolla, A., Jaworski, A., Larson, J. E., Jakupciak, J. P., Chuzhanova, N., Abeysinghe, S. S., et al. (2004). Breakpoints of gross deletions coincide with non-B DNA conformations. Proc. Natl. Acad. Sci. U.S.A. 101, 14162-14167. doi: 10.1073/pnas.0405974101

Bacolla, A., Tainer, J. A., Vasquez, K. M., and Cooper, D. N. (2016). Translocation and deletion breakpoints in cancer genomes are associated with potential non-B DNA-forming sequences. Nucleic Acids Res. 44, 5673-5688. doi: 10.1093/nar/gkw261

Bacolla, A., and Wells, R. D. (2004). Non-B DNA conformations, genomic rearrangements, and human disease. J. Biol. Chem. 279, 47411-47414. doi: 10.1074/jbc.R400028200

Bansal, M., Kumar, A., and Yella, V. R. (2014). Role of DNA sequence based structural features of promoters in transcription initiation and gene expression. Curr. Opin. Struct. Biol. 25, 77-85. doi: 10.1016/j.sbi.2014.01.007

Belotserkovskii, B. P., De Silva, E., Tornaletti, S., Wang, G., Vasquez, K. M., and Hanawalt, P. C. (2007). A triplex-forming sequence from the human c-MYC promoter interferes with DNA transcription. J. Biol. Chem. 282, 32433-32441. doi: 10.1074/jbc.M704618200

Bochman, M. L., Paeschke, K., and Zakian, V. A. (2012). DNA secondary structures: stability and function of G-quadruplex structures. Nat. Rev. Genet. 13, 770-780. doi: 10.1038/nrg3296

Boyer, A. S., Grgurevic, S., Cazaux, C., and Hoffmann, J. S. (2013). The human specialized DNA polymerases and non-B DNA: vital relationships to preserve genome integrity. J. Mol. Biol. 425, 4767-4781. doi: 10.1016/j.jmb.2013. 09.022

Caporale, L. H. (2003). Natural selection and the emergence of a mutation phenotype: an update of the evolutionary synthesis considering mechanisms that affect genome variation. Annu. Rev. Microbiol. 57, 467-485. doi: 10.1146/annurev.micro.57.030502.090855

Care, A., Cianetti, L., Giampaolo, A., Sposi, N. M., Zappavigna, V., Mavilio, F., et al. (1986). Translocation of c-myc into the immunoglobulin heavy-chain locus in human acute B-cell leukemia. A molecular analysis. EMBO J. 5, 905-911. of non-B DNA was calculated as the number of FOA resistant $\left(\mathrm{FOA}^{\mathrm{R}}\right)$ colonies divided by the number of total colonies.

\section{AUTHOR CONTRIBUTIONS}

GW, JZ, and KV designed the study; GW and JZ performed experiments; GW and $\mathrm{KV}$ analyzed data, discussed the results and wrote the manuscript.

\section{FUNDING}

This work was supported by an NIH/NCI grant to KV (CA093729).

\section{ACKNOWLEDGMENT}

We thank Dr. Catherine Freudenreich (Tufts University) for providing reagents and advice for the YAC screening system.

Cer, R. Z., Donohue, D. E., Mudunuri, U. S., Temiz, N. A., Loss, M. A., Starner, N. J., et al. (2013). Non-B DB v2.0: a database of predicted non-B DNAforming motifs and its associated tools. Nucleic Acids Res. 41, D94-D100. doi: 10.1093/nar/gks955

Chen, X., Shen, Y., Zhang, F., Chiang, C., Pillalamarri, V., Blumenthal, I., et al. (2013). Molecular analysis of a deletion hotspot in the NRXN1 region reveals the involvement of short inverted repeats in deletion CNVs. Am. J. Hum. Genet. 92, 375-386. doi: 10.1016/j.ajhg.2013.02.006

Choi, J., and Majima, T. (2011). Conformational changes of non-B DNA. Chem. Soc. Rev. 40, 5893-5909. doi: 10.1039/c1cs15153c

Cleary, M. L., and Sklar, J. (1985). Nucleotide sequence of a t $(14 ; 18)$ chromosomal breakpoint in follicular lymphoma and demonstration of a breakpoint-cluster region near a transcriptionally active locus on chromosome 18. Proc. Natl. Acad. Sci. U.S.A. 82, 7439-7443. doi: 10.1073/pnas.82.21.7439

De Muyt, A., Jessop, L., Kolar, E., Sourirajan, A., Chen, J., Dayani, Y., et al. (2012). BLM helicase ortholog Sgs1 is a central regulator of meiotic recombination intermediate metabolism. Mol. Cell 46, 43-53. doi: 10.1016/j.molcel.2012.02.020

DiCroce, P. A., and Krontiris, T. G. (1995). The BCL2 major breakpoint region is a sequence- and cell-cycle-specific binding site of the $\mathrm{Ku}$ antigen. Proc. Natl. Acad. Sci. U.S.A. 92, 10137-10141. doi: 10.1073/pnas.92.22. 10137

Du, X., Gertz, E. M., Wojtowicz, D., Zhabinskaya, D., Levens, D., Benham, C. J., et al. (2014). Potential non-B DNA regions in the human genome are associated with higher rates of nucleotide mutation and expression variation. Nucleic Acids Res. 42, 12367-12379. doi: 10.1093/nar/gku921

Frank-Kamenetskii, M. D., and Mirkin, S. M. (1995). Triplex DNA structures. Annu. Rev. Biochem. 64, 65-95. doi: 10.1146/annurev.bi.64.070195. 000433

Froelich-Ammon, S. J., Gale, K. C., and Osheroff, N. (1994). Site-specific cleavage of a DNA hairpin by topoisomerase II. DNA secondary structure as a determinant of enzyme recognition/cleavage. J. Biol. Chem. 269, 7719-7725.

Goldman, J. A., Garlick, J. D., and Kingston, R. E. (2010). Chromatin remodeling by imitation switch (ISWI) class ATP-dependent remodelers is stimulated by histone variant H2A.Z. J. Biol. Chem. 285, 4645-4651. doi: 10.1074/jbc.M109.072348

Haluska, F. G., Tsujimoto, Y., and Croce, C. M. (1988). The t(8;14) breakpoint of the EW 36 undifferentiated lymphoma cell line lies 5' of MYC in a region prone to involvement in endemic Burkitt's lymphomas. Nucleic Acids Res. 16, 2077-2085. doi: 10.1093/nar/16.5.2077 
Hanawalt, P. C., and Spivak, G. (2008). Transcription-coupled DNA repair: two decades of progress and surprises. Nat. Rev. Mol. Cell Biol. 9, 958-970. doi: $10.1038 / \mathrm{nrm} 2549$

Hausser, J., and Zavolan, M. (2014). Identification and consequences of miRNAtarget interactions-beyond repression of gene expression. Nat. Rev. Genet. 15, 599-612. doi: 10.1038/nrg3765

Jager, U., Bocskor, S., Le, T., Mitterbauer, G., Bolz, I., Chott, A., et al. (2000). Follicular lymphomas' BCL-2/IgH junctions contain templated nucleotide insertions: novel insights into the mechanism of $\mathrm{t}(14 ; 18)$ translocation. Blood 95, 3520-3529.

Jain, A., Wang, G., and Vasquez, K. M. (2008). DNA triple helices: biological consequences and therapeutic potential. Biochimie 90, 1117-1130. doi: 10.1016/j.biochi.2008.02.011

Joos, S., Haluska, F. G., Falk, M. H., Henglein, B., Hameister, H., Croce, C. M., et al. (1992). Mapping chromosomal breakpoints of Burkitt's $t(8 ; 14)$ translocations far upstream of c-myc. Cancer Res. 52, 6547-6552.

Kamat, M. A., Bacolla, A., Cooper, D. N., and Chuzhanova, N. (2016). A role for non-B DNA forming sequences in mediating microlesions causing human inherited disease. Hum. Mutat. 37, 65-73. doi: 10.1002/humu.22917

Kikin, O., D’Antonio, L., and Bagga, P. S. (2006). QGRS Mapper: a web-based server for predicting G-quadruplexes in nucleotide sequences. Nucleic Acids Res. 34, W676-W682. doi: 10.1093/nar/gkl253

Kovalchuk, A. L., Muller, J. R., and Janz, S. (1997). Deletional remodeling of c-myc-deregulating chromosomal translocations. Oncogene 15, 2369-2377. doi: 10.1038/sj.onc. 1201409

Krasilnikova, M. M., and Mirkin, S. M. (2004). Analysis of triplet repeat replication by two-dimensional gel electrophoresis. Methods Mol. Biol. 277, 19-28.

Lander, E. S., Linton, L. M., Birren, B., Nusbaum, C., Zody, M. C., Baldwin, J., et al. (2001). Initial sequencing and analysis of the human genome. Nature 409, 860-921. doi: 10.1038/35057062

Lane, A. N., Chaires, J. B., Gray, R. D., and Trent, J. O. (2008). Stability and kinetics of G-quadruplex structures. Nucleic Acids Res. 36, 5482-5515. doi: 10.1093/nar/gkn517

Latha, K. S., Anitha, S., Rao, K. S., and Viswamitra, M. A. (2002). Molecular understanding of aluminum-induced topological changes in (CCG) 12 triplet repeats: relevance to neurological disorders. Biochim. Biophys. Acta 1588, 5664. doi: 10.1016/S0925-4439(02)00133-3

Lewin, R. (1986). "Computer genome" is full of junk DNA. Science 232, 577-578. doi: 10.1126/science.3704643

Lobachev, K. S., Gordenin, D. A., and Resnick, M. A. (2002). The Mre11 complex is required for repair of hairpin-capped double-strand breaks and prevention of chromosome rearrangements. Cell 108, 183-193. doi: 10.1016/S00928674(02)00614-1

Lu, S., Wang, G., Bacolla, A., Zhao, J., Spitser, S., and Vasquez, K. M. (2015). Short inverted repeats are hotspots for genetic instability: relevance to cancer genomes. Cell Rep. 10, 1674-1680. doi: 10.1016/j.celrep.2015.02.039

Mankouri, H. W., Ashton, T. M., and Hickson, I. D. (2011). Holliday junctioncontaining DNA structures persist in cells lacking Sgs1 or Top3 following exposure to DNA damage. Proc. Natl. Acad. Sci. U.S.A. 108, 4944-4949. doi: $10.1073 /$ pnas. 1014240108

Mankouri, H. W., and Hickson, I. D. (2007). The RecQ helicase-topoisomerase IIIRmil complex: a DNA structure-specific 'dissolvasome'? Trends Biochem. Sci. 32, 538-546. doi: 10.1016/j.tibs.2007.09.009

Mefford, H. C., and Eichler, E. E. (2009). Duplication hotspots, rare genomic disorders, and common disease. Curr. Opin. Genet. Dev. 19, 196-204. doi: 10.1016/j.gde.2009.04.003

Michelotti, G. A., Michelotti, E. F., Pullner, A., Duncan, R. C., Eick, D., and Levens, D. (1996). Multiple single-stranded cis elements are associated with activated chromatin of the human c-myc gene in vivo. Mol. Cell. Biol. 16, 2656-2669. doi: 10.1128/MCB.16.6.2656

Mirkin, S. M. (2007). Expandable DNA repeats and human disease. Nature 447, 932-940. doi: 10.1038/nature05977

Mirkin, S. M., Lyamichev, V. I., Drushlyak, K. N., Dobrynin, V. N., Filippov, S. A., and Frank-Kamenetskii, M. D. (1987). DNA H form requires a homopurinehomopyrimidine mirror repeat. Nature 330, 495-497. doi: 10.1038/330495a0

Montoto, S., Lopez-Guillermo, A., Colomer, D., Esteve, J., Bosch, F., Ferrer, A., et al. (2003). Incidence and clinical significance of bcl-2/IgH rearrangements in follicular lymphoma. Leuk. Lymphoma 44, 71-76. doi: 10.1080/1042819021000050052

Moreau, S., Morgan, E. A., and Symington, L. S. (2001). Overlapping functions of the Saccharomyces cerevisiae Mre11, Exol and Rad27 nucleases in DNA metabolism. Genetics 159, 1423-1433.

Morillon, A., Karabetsou, N., O’Sullivan, J., Kent, N., Proudfoot, N., and Mellor, J. (2003). Isw1 chromatin remodeling ATPase coordinates transcription elongation and termination by RNA polymerase II. Cell 115, 425-435. doi: 10.1016/S0092-8674(03)00880-8

Parkinson, G. N., Lee, M. P., and Neidle, S. (2002). Crystal structure of parallel quadruplexes from human telomeric DNA. Nature 417, 876-880. doi: 10.1038 /nature755

Perera, R. J., and Ray, A. (2012). Epigenetic regulation of miRNA genes and their role in human melanomas. Epigenomics 4, 81-90. doi: 10.2217/epi.11.114

Pognan, F., and Paoletti, C. (1992). Does cruciform DNA provide a recognition signal for DNA-topoisomerase II? Biochimie 74, 1019-1023. doi: 10.1016/03009084(92)90022-7

Popescu, N. C. (2003). Genetic alterations in cancer as a result of breakage at fragile sites. Cancer Lett. 192, 1-17. doi: 10.1016/S0304-3835(02)00596-7

Raghavan, S. C., Swanson, P. C., Wu, X., Hsieh, C. L., and Lieber, M. R. (2004). A non-B-DNA structure at the Bcl-2 major breakpoint region is cleaved by the RAG complex. Nature 428, 88-93. doi: 10.1038/nature02355

Ramiro, A. R., Jankovic, M., Callen, E., Difilippantonio, S., Chen, H. T., McBride, K. M., et al. (2006). Role of genomic instability and p53 in AID-induced c-myc-Igh translocations. Nature 440, 105-109. doi: 10.1038/nature04495

Rich, A., and Zhang, S. (2003). Timeline: Z-DNA: the long road to biological function. Nat. Rev. Genet. 4, 566-572. doi: 10.1038/nrg1115

Rimokh, R., Rouault, J. P., Wahbi, K., Gadoux, M., Lafage, M., Archimbaud, E., et al. (1991). A chromosome 12 coding region is juxtaposed to the MYC protooncogene locus in a $\mathrm{t}(8 ; 12)(\mathrm{q} 24 ; \mathrm{q} 22)$ translocation in a case of B-cell chronic lymphocytic leukemia. Genes Chromosomes Cancer 3, 24-36. doi: $10.1002 /$ gcc. 2870030106

Saglio, G., Grazia Borrello, M., Guerrasio, A., Sozzi, G., Serra, A., di Celle, P. F., et al. (1993). Preferential clustering of chromosomal breakpoints in Burkitt's lymphomas and L3 type acute lymphoblastic leukemias with a $\mathrm{t}(8 ; 14)$ translocation. Genes Chromosomes Cancer 8, 1-7. doi: 10.1002/gcc.2870080102

Siddiqui-Jain, A., Grand, C. L., Bearss, D. J., and Hurley, L. H. (2002). Direct evidence for a G-quadruplex in a promoter region and its targeting with a small molecule to repress c-MYC transcription. Proc. Natl. Acad. Sci. U.S.A. 99, 11593-11598. doi: 10.1073/pnas.182256799

Sinclair, P. B., Parker, H., An, Q., Rand, V., Ensor, H., Harrison, C. J., et al. (2011). Analysis of a breakpoint cluster reveals insight into the mechanism of intrachromosomal amplification in a lymphoid malignancy. Hum. Mol. Genet. 20, 2591-2602. doi: 10.1093/hmg/ddr159

Sinden, R. R., Zheng, G. X., Brankamp, R. G., and Allen, K. N. (1991). On the deletion of inverted repeated DNA in Escherichia coli: effects of length, thermal stability, and cruciform formation in vivo. Genetics 129, 991-1005.

Smolle, M., Venkatesh, S., Gogol, M. M., Li, H., Zhang, Y., Florens, L., et al. (2012). Chromatin remodelers Isw1 and Chd1 maintain chromatin structure during transcription by preventing histone exchange. Nat. Struct. Mol. Biol. 19, 884-892. doi: 10.1038/nsmb.2312

Trujillo, K. M., and Sung, P. (2001). DNA structure-specific nuclease activities in the Saccharomyces cerevisiae Rad50*Mre11 complex. J. Biol. Chem. 276, 35458-35464. doi: 10.1074/jbc.M105482200

van Holde, K., and Zlatanova, J. (1994). Unusual DNA structures, chromatin and transcription. Bioessays 16, 59-68. doi: 10.1002/bies.950160110

Vasquez, K. M., and Wang, G. (2013). The yin and yang of repair mechanisms in DNA structure-induced genetic instability. Mutat. Res. 74, 118-131. doi: 10.1016/j.mrfmmm.2012.11.005

Veitia, R. A., and Bottani, S. (2009). Whole genome duplications and a 'function' for junk DNA? Facts and hypotheses. PLoS ONE 4:e8201. doi: 10.1371/journal.pone.0008201

Venkatesan, R. N., Bielas, J. H., and Loeb, L. A. (2006). Generation of mutator mutants during carcinogenesis. DNA Repair (Amst) 5, 294-302. doi: 10.1016/j.dnarep.2005.10.012

Voineagu, I., Narayanan, V., Lobachev, K. S., and Mirkin, S. M. (2008). Replication stalling at unstable inverted repeats: interplay between DNA hairpins and 
fork stabilizing proteins. Proc. Natl. Acad. Sci. U.S.A. 105, 9936-9941. doi: 10.1073/pnas.0804510105

Voloshin, O. N., Mirkin, S. M., Lyamichev, V. I., Belotserkovskii, B. P., and Frank-Kamenetskii, M. D. (1988). Chemical probing of homopurinehomopyrimidine mirror repeats in supercoiled DNA. Nature 333, 475-476. doi: $10.1038 / 333475 \mathrm{a} 0$

Wang, G., Carbajal, S., Vijg, J., DiGiovanni, J., and Vasquez, K. M. (2008). DNA structure-induced genomic instability in vivo. J. Natl. Cancer Inst. 100, 18151817. doi: 10.1093/jnci/djn385

Wang, G., Christensen, L. A., and Vasquez, K. M. (2006). Z-DNAforming sequences generate large-scale deletions in mammalian cells. Proc. Natl. Acad. Sci. U.S.A. 103, 2677-2682. doi: 10.1073/pnas.05110 84103

Wang, G., Gaddis, S., and Vasquez, K. M. (2013). Methods to detect replication-dependent and replication-independent DNA structureinduced genetic instability. Methods 64, 67-72. doi: 10.1016/j.ymeth.2013. 08.004

Wang, G., and Vasquez, K. M. (2004). Naturally occurring H-DNA-forming sequences are mutagenic in mammalian cells. Proc. Natl. Acad. Sci. U.S.A. 101, 13448-13453. doi: 10.1073/pnas.0405116101

Wang, G., and Vasquez, K. M. (2006). Non-B DNA structure-induced genetic instability. Mutat. Res. 598, 103-119. doi: 10.1016/j.mrfmmm.2006. 01.019

Wang, G., and Vasquez, K. M. (2009). Models for chromosomal replicationindependent non-B DNA structure-induced genetic instability. Mol. Carcinog. 48, 286-298. doi: 10.1002/mc.20508

Wang, G., and Vasquez, K. M. (2014). Impact of alternative DNA structures on DNA damage, DNA repair, and genetic instability. DNA Repair (Amst) 19, 143-151. doi: 10.1016/j.dnarep.2014.03.017

Wang, G., Zhao, J., and Vasquez, K. M. (2009). Methods to determine DNA structural alterations and genetic instability. Methods 48, 54-62. doi: 10.1016/j.ymeth.2009.02.012

Wang, J. C. (2002). Cellular roles of DNA topoisomerases: a molecular perspective. Nat. Rev. Mol. Cell Biol. 3, 430-440. doi: 10.1038/nrm831
Wells, R. D., Dere, R., Hebert, M. L., Napierala, M., and Son, L. S. (2005). Advances in mechanisms of genetic instability related to hereditary neurological diseases. Nucleic Acids Res. 33, 3785-3798. doi: 10.1093/nar/gki697

Wilda, M., Busch, K., Klose, I., Keller, T., Woessmann, W., Kreuder, J., et al. (2004). Level of MYC overexpression in pediatric Burkitt's lymphoma is strongly dependent on genomic breakpoint location within the MYC locus. Genes Chromosomes Cancer 41, 178-182. doi: 10.1002/gcc.20063

Wittig, B., Wolfl, S., Dorbic, T., Vahrson, W., and Rich, A. (1992). Transcription of human c-myc in permeabilized nuclei is associated with formation of Z-DNA in three discrete regions of the gene. EMBO J. 11, 4653-4663.

Wolfl, S., Wittig, B., and Rich, A. (1995). Identification of transcriptionally induced Z-DNA segments in the human c-myc gene. Biochim. Biophys. Acta 1264, 294-302. doi: 10.1016/0167-4781(95)00155-7

Wreesmann, V. B., and Singh, B. (2005). Chromosomal aberrations in squamous cell carcinomas of the upper aerodigestive tract: biologic insights and clinical opportunities. J. Oral Pathol. Med. 34, 449-459. doi: 10.1111/j.16000714.2005.00343.x

Wyatt, R. T., Rudders, R. A., Zelenetz, A., Delellis, R. A., and Krontiris, T. G. (1992). BCL2 oncogene translocation is mediated by a chi-like consensus. J. Exp. Med. 175, 1575-1588. doi: 10.1084/jem.175.6.1575

Zhao, J., Bacolla, A., Wang, G., and Vasquez, K. M. (2010). Non-B DNA structureinduced genetic instability and evolution. Cell. Mol. Life Sci. 67, 43-62. doi: 10.1007/s00018-009-0131-2

Conflict of Interest Statement: The authors declare that the research was conducted in the absence of any commercial or financial relationships that could be construed as a potential conflict of interest.

Copyright (C) 2016 Wang, Zhao and Vasquez. This is an open-access article distributed under the terms of the Creative Commons Attribution License (CC BY). The use, distribution or reproduction in other forums is permitted, provided the original author(s) or licensor are credited and that the original publication in this journal is cited, in accordance with accepted academic practice. No use, distribution or reproduction is permitted which does not comply with these terms. 